\title{
Dietas para cerdos en iniciación incluyendo salvado de trigo y adicionadas con xilanasas
}

\section{Starting pig diets including wheat bran and supplemented with xylanase}

\author{
José Alfredo Martínez-Aispuro ${ }^{1}$, José Luis Figueroa-Velasco ${ }^{1 *}$, José Luis Cordero-Mora ${ }^{1}$, María Teresa \\ Sánchez-Torres-Esqueda ${ }^{1}$, Manuel Martínez-Aispuro ${ }^{2}$ \\ ${ }^{1}$ Programa de Ganadería, Campus Montecillo, Colegio de Postgraduados. Km. 36.5 Carretera México-Texcoco. CP. 56230, \\ Montecillo, Texcoco, Estado de México, México \\ ${ }^{2}$ Nutreco. Trouw Nutrition. Parque Industrial Belenes Norte, CP. 45150, Zapopan, Jalisco, México \\ ${ }^{*}$ Autor de correspondencia: jlfigueroa@colpos.mx
}

Artículo científico recibido: 06 de enero de 2016, aceptado: 06 de abril de 201

RESUMEN. El objetivo fue evaluar la adición de xilanasas en dietas con alta cantidad de salvado de trigo (ST) y dos niveles de energía para cerdos en iniciación sobre la respuesta productiva, características de la canal y concentración de urea en plasma. Se utilizaron 48 cerdos híbridos castrados de $7.98 \mathrm{~kg}$ de peso inicial y $28 \mathrm{~d}$ de edad, distribuidos en un diseño completamente al azar. Los tratamientos ( $\mathrm{T}$ ) fueron: T1, dieta testigo: $3350 \mathrm{Kcal}$ de $\mathrm{EM} \mathrm{kg}^{-1}$; T2: 3275 Kcal de $\mathrm{EM} \mathrm{kg}^{-1}$ + xilanasas; T3: $3350 \mathrm{Kcal}$ de EM kg-1 + xilanasas; T4: $3350 \mathrm{Kcal}^{-1 e} \mathrm{EM} \mathrm{kg}^{-1}+\mathrm{ST}$ + xilanasas; T5: $3275 \mathrm{Kcal}$ de $\mathrm{EM} \mathrm{kg}{ }^{-1}+\mathrm{ST}$ + xilanasas; T6: 3350 de $\mathrm{EM} \mathrm{kg}^{-1}+\mathrm{ST}+$ xilanasas. Los datos se analizaron con ANOVA, las medias de los tratamientos se compararon con la prueba de Tukey $(p \leq 0.1)$. Para la ganancia de peso (GDP), consumo de alimento, peso final (PVF) y ganancia de carne magra (GCM) se observó mejor respuesta en cerdos alimentados $\sin \mathrm{ST}(\mathrm{p} \leq 0.1)$; compensando las xilanasas la reducción de energía. La inclusión de xilanasas en dietas con ST redujo la GDP y el pVF ( $\leq \leq 0.1)$. La inclusión de ST no modificó la grasa dorsal (GD), área del músculo longissimus (AML) y GCM ( $p>0.1$ ). La GD y AML no fueron afectadas al reducir la energía en las dietas sin ST $(p>0.1)$. Las dietas con alto contenido de salvado de trigo afectan las variables productivas; aunque se compensa con la adición de xilanasas mientras se respete la matriz energética.

Palabras clave: Energía, polisacáridos no almidonosos, arabinoxilanos, enzimas, fibra

ABSTRACT. The objective was to evaluate the addition of xylanase in nursery pig diets with a high amount of wheat bran (WB) and two energy levels. Growth performance, carcass characteristics and plasma urea concentration were assessed. Forty-eight hybrid barrows were used $(7.98 \mathrm{~kg}$ initial body weight and $28 \mathrm{~d}$ of age), allotted in a completely randomized design. Treatments (T) were: T1, control diet: $3350 \mathrm{Kcal}$; T2: $3275 \mathrm{Kcal}+$ xylanase; T3: 3350 Kcal + xylanase; T4: $3350 \mathrm{Kcal}+\mathrm{WB}+$ xylanase; T5: $3275 \mathrm{Kcal}+\mathrm{WB}+$ xylanase; T6: 3350 + WB + xylanase. The variables were analyzed with ANOVA and treatment means were compared with Tukey's test $(\mathrm{p} \leq 0.1)$. For average daily gain (ADG), average daily feed intake, final body weight and fat free lean gain (FFLG), better response was observed in pigs fed diets without WB $(p \leq 0.1)$ where xylanase compensated energy reduction. The inclusion of xylanase in WB diets reduced the ADG and final body weight $(p \leq 0.1)$. Supplementation with WB did not change backfat thickness, longissimus muscle area (LMA) and lean meat percentage $(p>0.1)$. The backfat thickness and LMA were not affected ( $p>0.1)$ by reducing energy in diets without WB $(p>0.1)$. In conclusion, diets high in wheat bran content affect growth performance variables, but it is offset by the addition of xylanase, as long as the energy matrix is observed.

Key words: Energy, non-starch polysaccharides, arabinoxylans, enzymes, fiber 
Martínez- Aispuro et al. Xilanasas en dietas para cerdos en iniciación 4(10):73-80,2017

\section{INTRODUCCIÓN}

El salvado de trigo es un subproducto en la producción de la harina de trigo que utiliza en la dieta de los cerdos (Hassan et al. 2008). Este subproducto se caracteriza por su alto nivel de fibra la cual es extremadamente resistente a la degradación en el tracto gastrointestinal (Noblet y Le Goff 2001). El uso de la fibra en las dietas para cerdos se ha restringido debido a que a medida que se incrementa, la digestibilidad de los demás nutrientes y los parámetros productivos se afectan (Xu et al. 2010). Sin embargo, ingredientes con alto contenido de fibra (polisacáridos no almidonosos, PNA), como el salvado de trigo, se han incorporado en alimentos para cerdos recién destetados, ya que mejora la salud intestinal, modula la actividad y composición de la microflora intestinal (Molist et al. 2011). Además, las bacterias pueden fermentar la fibra en el intestino grueso produciendo la liberación de ácidos grasos volátiles que sirven como energía (Jha y Leterme 2012).

Debido a que los cerdos no producen la cantidad suficiente de enzimas para romper las cadenas de PNA, una opción la utilización de nutrientes ricos de ingredientes en este tipo de polisacáridos es la suplementación enzimática (Zijlstra et al. 2010).Ya que el salvado de trigo contiene una alta cantidad de PNA (arabinoxilanos) (Saha 2003, Rosenfelder et al. 2013), el uso enzimas celulolíticas (xilanasas) puede representar una excelente opción en la alimentación de cerdos al destete, ya que se ha observado que mejora la digestibilidad ileal aparente y total, la digestibilidad de la proteína y los carbohidratos en dietas de cerdos en crecimiento con la inclusión de salvado de trigo (Yin et al. 2000, Nortey et al. 2008). Por lo que el uso de xilanasas puede potencializar el uso de los nutrientes del salvado de trigo y a su vez gozar de los beneficios de dietas altas en fibra, favoreciendo la rápida adaptación de cerdos recién destetados al nuevo tipo de alimentación, reflejándose en un mejor comportamiento productivo. Por tal motivo, el objetivo de esta investigación fue probar la adición de xilanasas en dietas con alto contenido de salvado de trigo y dos niveles de energía para cerdos en iniciación sobre las variables productivas, las características de la canal y la concentración de urea en plasma.

\section{MATERIALES Y MÉTODOS}

Los procedimientos experimentales se realizaron de acuerdo con las recomendaciones del International Guiding Principles for Biomedical Research Involving Animals (CIOMS 2002) y con la norma mexicana (NOM-062-Z00-1999) para el uso de animales en experimentación (SAGARPA 2001).

El estudio se realizó en la Unidad Porcina de la Granja Experimental del Colegio de Postgraduados, en Montecillo, Estado de México, localizada a $98^{\circ} 48^{\prime} 27^{\prime \prime}$ LO y a $19^{\circ} 48^{\prime} 23^{\prime \prime}$ LN a una altitud de $2241 \mathrm{~m}$. Con clima templado subhúmedo con lluvias en verano, temperatura media anual de 15.2 ${ }^{\circ} \mathrm{C}$ y precipitación media anual de $644.8 \mathrm{~mm}$ (García 1988).

En el experimento se evaluaron dos concentraciones de energía metabolizable (EM) y dos concentraciones de salvado de trigo con o sin la adición de xilanasas de acuerdo con la cantidad señalada por la Casa comercial (DANISCO ${ }^{\circledR}$ ) para cerdos en etapa de iniciación. Uno de los niveles de energía establecidos fue el recomendado por el NRC (2012) y el otro se estableció respetando la matriz energética de las xilanasas determinada y recomendada por la casa comercial, ya que se incrementa la disponibilidad de $75 \mathrm{Kcal}$ de EM en dietas para cerdos. Los tratamientos $(\mathrm{T})$ fueron los siguientes: T1, dieta testigo (EM: $3300 \mathrm{Kcal} \mathrm{kg}^{-1}$, sin salvado de trigo; T2, (EM: $3225 \mathrm{Kcal} \mathrm{kg}^{-1}$, sin salvado de trigo, xilanasas (0.005\%); T3, (EM: 3300

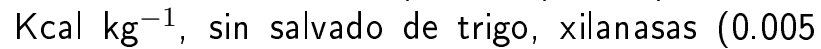
\%); T4, (EM: $3300 \mathrm{Kcal} \mathrm{kg}^{-1}$, salvado de trigo: 6 $\%$; T5, (EM: $3225 \mathrm{Kcal} \mathrm{kg}^{-1}$, salvado de trigo:6\%, xilanasas (0.005\%); T6, (EM: $3300 \mathrm{Kcal} \mathrm{kg}^{-1}$, salvado de trigo: $6 \%$, xilanasas $(0.005 \%)$. Las dietas fueron formuladas con comando Solver de Excel para cubrir o exceder los requerimientos sugeridos por el NRC (2012) con excepción de la energía (Tabla 1).

Las unidades experimentales fueron 48 cerdos 
Tabla 1. Dietas experimentales para cerdos en crecimiento.

\begin{tabular}{|c|c|c|c|c|c|c|}
\hline & \multicolumn{6}{|c|}{ Tratamientos } \\
\hline Ingrediente (\%) & T1 & T2 & T3 & $\bar{T} 4$ & T5 & T6 \\
\hline Sorgo & 65.05 & 66.68 & 65.05 & 58.93 & 60.56 & 58.93 \\
\hline Pasta de soya & 29.22 & 28.91 & 29.22 & 28.23 & 27.92 & 28.23 \\
\hline Salvado de trigo & 0.00 & 0.00 & 0.00 & 6.00 & 6.00 & 6.00 \\
\hline Aceite de soya & 1.32 & 0.51 & 1.84 & 2.97 & 1.64 & 2.97 \\
\hline L-Lisina $(50 \%)$ & 0.61 & 0.62 & 0.61 & 0.63 & 0.64 & 0.63 \\
\hline DL-Metionina & 0.17 & 0.17 & 0.17 & 0.18 & 0.17 & 0.18 \\
\hline L-Triptófano & 0.03 & 0.03 & 0.03 & 0.03 & 0.03 & 0.03 \\
\hline L-Treonina & 0.15 & 0.15 & 0.15 & 0.16 & 0.16 & 0.16 \\
\hline Premezcla de vitaminas ${ }^{A}$ & 0.15 & 0.15 & 0.15 & 0.15 & 0.15 & 0.15 \\
\hline Premezcla de minerales ${ }^{B}$ & 0.15 & 0.15 & 0.15 & 0.15 & 0.15 & 0.15 \\
\hline Xilanasas & 0.00 & 0.005 & 0.005 & 0.000 & 0.005 & 0.005 \\
\hline Fitasa & 0.01 & 0.01 & 0.01 & 0.01 & 0.01 & 0.01 \\
\hline Sal común & 0.49 & 0.49 & 0.49 & 0.49 & 0.49 & 0.49 \\
\hline $\mathrm{CaCO} 3$ & 1.32 & 1.32 & 1.32 & 1.32 & 1.32 & 1.32 \\
\hline Ortofosfato de calcio & 0.80 & 0.80 & 0.80 & 0.76 & 0.76 & 0.76 \\
\hline \multicolumn{7}{|l|}{ Contenido calculado: } \\
\hline Energía metabolizable, Mcal kg ${ }^{-1}$ ) & 3.300 & 3.225 & 3.300 & 3.300 & 3.225 & 3300 \\
\hline Proteína cruda & 20.00 & 20.00 & 20.00 & 20.00 & 20.00 & 20.00 \\
\hline Calcio & 0.73 & 0.73 & 0.73 & 0.73 & 0.73 & 0.73 \\
\hline Fósforo & 0.56 & 0.56 & 0.56 & 0.60 & 0.56 & 0.56 \\
\hline Lisina & 1.18 & 1.18 & 1.18 & 1.18 & 1.18 & 1.18 \\
\hline Treonina & 0.74 & 0.74 & 0.74 & 0.74 & 0.74 & 0.74 \\
\hline Triptc & 0.23 & 0.23 & 0.23 & 0.23 & 0.23 & 0.23 \\
\hline Metionina & 0.43 & 0.43 & 0.43 & 0.43 & 0.43 & 0.43 \\
\hline \multicolumn{7}{|l|}{ Análisis determinado } \\
\hline Proteína cruda & 19.89 & 19.86 & 19.84 & 19.94 & 19.67 & 19.77 \\
\hline Calcio & 0.68 & 0.67 & 0.70 & 0.72 & 0.69 & 0.68 \\
\hline Fósforo & 0.50 & 0.54 & 0.48 & 0.51 & 0.53 & 0.52 \\
\hline Costo $(\$ \mathrm{~kg})^{C}$ & 5.40 & 5.23 & 5.40 & 5.53 & 5.36 & 5.53 \\
\hline \multicolumn{7}{|c|}{$\begin{array}{l}\text { A Proporcionó por kg de alimento: vitamina } \mathrm{A}, 15,000 \mathrm{UI} \text {; vitamina } \mathrm{D} 3,2,500 \mathrm{UI} \text {; vitamina } \\
\mathrm{E}, 37.5 \mathrm{UI} \text {; vitamina } \mathrm{K}, 2.5 \mathrm{mg} \text {; tiamina, } 2.25 \mathrm{mg} \text {; riboflavina, } 6.25 \mathrm{mg} \text {; niacina, } 50 \mathrm{mg} \text {; } \\
\text { piridoxina, } 2.5 \mathrm{mg} \text {; cianocobalamina, } 0.0375 \mathrm{mg} \text {; biotina, } 0.13 \mathrm{mg} \text {; cloruro de colina, } 563 \mathrm{mg} \text {; } \\
\text { ácido pantoténico, } 20 \mathrm{mg} \text {; ácido fólico, } 1.25 \mathrm{mg} \text {. }{ }^{B} \text { Aportó por kg de alimento: Fe, } 150 \mathrm{mg} \text {; } \\
\mathrm{Zn}, 150 \mathrm{mg} \text {; Mn, } 150 \mathrm{mg} \text {; Cu, } 10 \mathrm{mg} \text {; Se, } 0.15 \mathrm{mg} \text {; I, } 0.9 \mathrm{mg} \text {; } \mathrm{Cr}, 0.2 \mathrm{mg} \text {. C Calculado con } \\
\text { base en el precio en pesos mexicanos de los ingredientes vigentes de febrero de } 2015 \text {. }\end{array}$} \\
\hline
\end{tabular}

(machos castrados) híbridos (Landrace $\times$ Yorkshire $\times$ Duroc), con peso vivo inicial (PVI) promedio de $7.98 \mathrm{~kg}$ y $28 \mathrm{~d}$ de edad. El diseño experimental fue completamente al azar con seis tratamientos y ocho repeticiones (un cerdo por repetición). Los cerdos se alojaron en corrales individuales equipados con comedero tipo tolva y bebedero de chupón. El alimento y el agua se ofrecieron a libre acceso. El periodo de evaluación fue de $21 \mathrm{~d}$.

La proteína cruda se determinó por el método de macrokjeldahl (AOAC 1990). La concentración de calcio y fósforo por espectrometría, en un espectrofotómetro de absorción atómica Perkin Elmer 4000, serie Lambda 2 (Karl et al. 1979). Al final del experimento se obtuvieron muestras de sangre de la vena cava anterior con tubos Vacutainer ${ }^{R}$ con heparina, los cuales se colocaron en hielo hasta centrifugarse en una centrifuga SIGMA 2-16k a $2500 \mathrm{~g}$ por $20 \mathrm{~min}$, para separar el plasma del paquete celular. El plasma se transfirió a tubos de polipropileno y se almacenó en un congelador SANYO MDF-436 a $-20{ }^{\circ} \mathrm{C}$ hasta la determinación de urea en plasma (Chaney y Marbach 1962).

Las variables de respuesta fueron consumo de alimento ( $C A L)$, ganancia diaria de peso (GDP), conversión alimenticia (CA), ganancia de carne magra (GCM) y peso vivo de los cerdos final (PVF), grasa dorsal (GD), porcentaje de carne magra (PCM), área del músculo Longissimus dorsi (AML) y concentración de urea en plasma (CUP). La GD y AML se midieron con un ultrasonido de tiempo real SonoVet 600 marca Medison, al inicio y final 
del experimento. Con estos datos y con los PVI y PVF se calculó la GCM y el PCM con la ecuación del NPCC (1991).

Con los datos se realizó una ANOVA mediante el procedimiento GLM, las medias de los tratamientos se compararon utilizando contrastes $(\mathrm{p} \leq 0.1)$ (SAS, 2010). El peso inicial se utilizó como covariable debido a la heterogeneidad de peso observada en los animales.

\section{RESULTADOS}

Para las variables productivas se presentan en la Tabla 2. No se detectaron diferencias ( $p>$ 0.1 ) entre tratamientos para la conversión alimenticia por efecto del uso de salvado de trigo, enzimas y la reducción del nivel de energía. Para la GDP, CAL y PVF se observó una mejor respuesta para los cerdos alimentados sin salvado de trigo $(p \leq 0.1)$.

La adición de xilanasas en las dietas sin salvado de trigo no tuvo efecto en las variables productivas cuando se utilizaron $3300 \mathrm{kcal}$ de EM ( $\mathrm{p}$ $>0.05$ ), aunque el uso de las enzimas compensó la reducción de la energía cuando se redujo en $75 \mathrm{kcal}$. Sin embargo la inclusión de xilanasas en dietas con salvado de trigo redujeron la GDP y el PVF ( $p \leq$ 0.08 ), de forma independiente del nivel de energía. La GCM disminuyó al utilizar xilanasas $(\mathrm{p} \leq 0.05)$ y al incrementar el contenido de salvado de trigo en la dieta $(p=0.09)$, efecto que se agudizó al reducir el nivel de energía $(p \leq 0.05)$.

Para las características de la canal (Tabla 3) la inclusión de salvado de trigo no afectó a ninguna de las variables (GDF, AMLF y PCMF) $(p>0.1)$. El uso de enzimas disminuyó el AMLF $(p \leq 0.05)$ e incrementó la GDF ( $p \leq 0.06)$, aunque no tuvo efecto sobre el PCMF ( $p>0.1)$. Para los tratamientos sin salvado de trigo la GDF se incrementó al adicionar las xilanasas ( $p \leq 0.1$ ); mientras que el PCMF se redujo al utilizar las enzimas $(p \leq 0.1)$ y reducir la cantidad de energía ( $p \leq 0.01$ ). El AMLF de las dietas sin salvado de trigo disminuyó $(p \leq 0.05)$ por el uso de enzimas. La GDF de las dietas con salvado de trigo incrementó $(p \leq 0.06)$ al utilizar xilanasas, mientras que la reducción de energía con el uso de enzimas no afecto la variable $(\mathrm{p}>0.1)$, mientras que el $A M L$ se redujo al disminuir la energía ( $p \leq$ 0.03). La GD y el AML no fueron afectadas ( $p>$ $0.1)$ por la reducción de energía $(-75 \mathrm{kcal})$ en las dietas sin salvado de trigo adicionadas con xilanasas. La concentración de urea en plasma fue mayor ( $p$ $\leq 0.04$ ) en los cerdos adicionados con xilanasas y sin salvado de trigo (Tabla 2).

\section{DISCUSIÓN}

La reducción en la GDP se relaciona con un menor CAL, debido a la alta concentración de salvado de trigo en la dieta, lo que se refleja en un menor PVF. Esto se atribuye a que el aumento de fibra dietaría por la inclusión de salvado de trigo reduce el consumo de alimento debido al alto contenido de PNA, ya que aumentan la viscosidad del tracto superior intestinal, reduciendo el vaciado estomacal y la velocidad de tránsito (Noblet y Le Goff 2001). Además la digestibilidad de la proteína, energía y materia seca disminuyen; trayendo como consecuencia una reducción de la GDP y el CAL, por lo que aumenta la CA (Xu et al. 2010, Ziemer et al. 2012).

La adición de xilanasas compensó la reducción de energía, tanto en dietas con salvado y sin salvado de trigo, por lo que pudo incrementarse la disponibilidad de energía en cerdos alimentados con una alta cantidad de fibra. En virtud de que los arabinoxilanos representan el mayor porcentaje de polisacáridos no amiláceos, lugar de acción de las xilanasas (Nortey et al. 2008). También se sabe que el uso de xilanasas en dietas a base de sorgo compensa la reducción de energía hasta en $105 \mathrm{kcal}$ de EM (Martínez-Aispuro et al. 2015), ingrediente base en las dietas sin y con salvado de trigo del presente trabajo.

Aunque no hubo un mejor comportamiento en las variables productivas para los tratamientos con más energía, se detectó una mayor acumulación de GD. El incremento de energía, y potencialmente de PC reflejado por una mayor CUP pudo traer como consecuencia un aumento de la GD y reducción del AML; ya que el uso de xilanasas potencializa la uti- 
Tabla 2. Comportamiento productivo de cerdos en iniciación, alimentados con una alta cantidad de salvado de trigo y suplementados con xilanasas.

\begin{tabular}{|c|c|c|c|c|c|c|c|c|c|c|}
\hline \multirow[t]{2}{*}{ Trat } & \multicolumn{2}{|c|}{ Salvado EM } & \multicolumn{3}{|c|}{ Xilanasas } & \multicolumn{3}{|c|}{ Comportamiento productivo } & \multirow[b]{2}{*}{ GCM (Kg) } & \multirow[b]{2}{*}{$\begin{array}{c}\text { CUP } \\
\mathrm{mg} \mathrm{dL}^{-1}\end{array}$} \\
\hline & $(\%)$ & $\begin{array}{l}\mathrm{Kcal} \\
\mathrm{Kg}^{-1}\end{array}$ & & $\begin{array}{c}\text { GDP }) \\
\left(\mathrm{Kg} \mathrm{d}^{-1}\right.\end{array}$ & $\begin{array}{c}\text { CAL }(\mathrm{Kg} \\
\left.\mathrm{d}^{-1}\right)\end{array}$ & CA & PVI (Kg) & PVF (Kg) & & \\
\hline 1 & 0 & 3300 & 0.000 & $0.78 \pm 0.02$ & $1.46 \pm 0.06$ & $1.87 \pm 0.08$ & $7.92 \pm 1.17$ & $23.53 \pm 0.59$ & $0.22 \pm 0.01$ & $10.52 \pm 1,36$ \\
\hline 2 & 0 & 3225 & 0.005 & $0.79 \pm 0.02$ & $1.52 \pm 0.06$ & $1.92 \pm 0.08$ & $8.03 \pm 1.17$ & $23.86 \pm 0.59$ & $0.21 \pm 0.01$ & $16.16 \pm 1,36$ \\
\hline 3 & 0 & 3300 & 0.005 & $0.77 \pm 0.03$ & $1.51 \pm 0.06$ & $1.94 \pm 0.09$ & $7.12 \pm 1.24$ & $23.54 \pm 0.63$ & $0.21 \pm 0.01$ & $15.21 \pm 1,38$ \\
\hline 4 & 6 & 3300 & 0.000 & $0.78 \pm 0.16$ & $1.39 \pm 0.06$ & $1.78 \pm 0.08$ & $7.81 \pm 1.24$ & $23.60 \pm 0.63$ & $0.22 \pm 0.01$ & $8.64 \pm 1,55$ \\
\hline 5 & 6 & 3220 & 0.005 & $0.71 \pm 0.03$ & $1.37 \pm 0.06$ & $1.89 \pm 0.09$ & $8.69 \pm 1.24$ & $22.27 \pm 0.63$ & $0.18 \pm 0.01$ & $12.84 \pm 1,55$ \\
\hline 6 & 6 & 3300 & 0.005 & $0.70 \pm 0.20$ & $1.33 \pm 0.06$ & $1.89 \pm 0.08$ & $8.36 \pm 1.17$ & $22.18 \pm 0.59$ & $0.18 \pm 0.01$ & $11.35 \pm 1,55$ \\
\hline \multicolumn{11}{|c|}{ Significancia $(P)$ de los contrastes } \\
\hline \multicolumn{4}{|c|}{ DSST vs DCST } & 0.05 & 0.01 & 0.41 & & 0.06 & 0.09 & 0.04 \\
\hline \multicolumn{4}{|c|}{ Trat 4 vs Trat 5 y 6} & 0.08 & 0.55 & 0.33 & & 0.08 & 0.006 & 0.03 \\
\hline \multicolumn{4}{|c|}{ Trat 4 vs Trat 5} & 0.10 & 0.45 & 0.80 & & 0.11 & 0.01 & 0.05 \\
\hline \multicolumn{4}{|c|}{ Trat 4 vs Trat 6} & 0.15 & 0.76 & 0.41 & & 0.14 & 0.02 & 0.06 \\
\hline \multicolumn{4}{|c|}{ Usar enzima } & 0.24 & 0.91 & 0.27 & & 0.26 & 0.01 & 0.04 \\
\hline
\end{tabular}

Trat = Tratamiento, GDP = Ganancia diaria de peso, CAL = Consumo de alimento, CA = Conversión alimenticia, PVI = Peso vivo inicial, PVF = Peso Vivo final, GCM = Ganancia de carne magra. CUP $=$ Concentración de urea en plasma. DSST $=$ Dietas sin salvado de trigo, DCST $=$ Dietas con salvado de trigo.

Tabla 3. Características de la canal y concentración de urea en plasma de cerdos en iniciación, alimentados con una alta cantidad de salvado de trigo y suplementados con xilanasas.

\begin{tabular}{|c|c|c|c|c|c|c|c|c|c|}
\hline \multirow[t]{2}{*}{ Trat } & \multirow{2}{*}{$\begin{array}{l}\text { Salvado } \\
\text { de trigo } \\
(\%)\end{array}$} & \multirow{2}{*}{$\begin{array}{l}\text { Energía } \\
\left(\mathrm{Kcal}^{-1} \mathrm{~kg}^{-1}\right)\end{array}$} & \multicolumn{3}{|c|}{ Xilanasas } & \multicolumn{2}{|c|}{ Características de la canal } & \multirow[b]{2}{*}{ PCMI } & \multirow[b]{2}{*}{ PCMF } \\
\hline & & & $(\%)$ & $\mathrm{GDI}(\mathrm{mm})$ & GDF (mm) & $\operatorname{AMLI}\left(\mathrm{cm}^{2}\right)$ & $\operatorname{AMLF}\left(\mathrm{cm}^{2}\right)$ & & \\
\hline 1 & 0.0 & 3300 & 0.000 & $2.67 \pm 0.12$ & $3.11 \pm 0.17$ & $8.75 \pm 0.29$ & $14.43 \pm 0.46$ & $52.60 \pm 0.66$ & $45.37 \pm 0.16$ \\
\hline 2 & 0.0 & 3225 & 0.005 & $2.55 \pm 0.12$ & $3.22 \pm 0.17$ & $8.54 \pm 0.29$ & $13.28 \pm 0.46$ & $52.66 \pm 0.66$ & $44.54 \pm 0.16$ \\
\hline 3 & 0.0 & 3300 & 0.005 & $2.33 \pm 0.13$ & $4.22 \pm 0.19$ & $8.10 \pm 0.31$ & $12.53 \pm 0.49$ & $51.72 \pm 0.70$ & $43.61 \pm 0.19$ \\
\hline 4 & 6.0 & 3300 & 0.000 & $2.51 \pm 0.13$ & $3.15 \pm 0.19$ & $8.00 \pm 0.31$ & $14.14 \pm 0.49$ & $51.08 \pm 0.70$ & $44.62 \pm 0.19$ \\
\hline 5 & 6.0 & 3225 & 0.005 & $2.42 \pm 0.13$ & $3.88 \pm 0.19$ & $8.13 \pm 0.31$ & $12.57 \pm 0.49$ & $51.99 \pm 0.70$ & $44.46 \pm 0.19$ \\
\hline 6 & 6.0 & 3300 & 0.005 & $2.63 \pm 0.12$ & $4.36 \pm 0.17$ & $8.92 \pm 0.29$ & $13.19 \pm 0.46$ & $52.71 \pm 0.66$ & $44.83 \pm 0.16$ \\
\hline \multicolumn{10}{|c|}{ Significancia $(P)$ de los contrastes } \\
\hline \multicolumn{5}{|c|}{ DSST vs DCST } & 0.19 & & 0.43 & & 0.74 \\
\hline \multicolumn{5}{|c|}{ Trat 1 vs Trat 2} & 0.46 & & 0.40 & & 0.01 \\
\hline \multicolumn{5}{|c|}{ Trat 1 vs Trat 3} & 0.07 & & 0.01 & & 0.01 \\
\hline \multicolumn{5}{|c|}{ Trat 2 vs Trat 3} & 0.07 & & 0.05 & & 0.17 \\
\hline \multicolumn{5}{|c|}{ Trat 4 vs Trat 5} & 0.06 & & 0.03 & & 0.82 \\
\hline \multicolumn{5}{|c|}{ Trat 4 vs Trat 6} & 0.06 & & 0.17 & & 0.75 \\
\hline \multicolumn{5}{|c|}{ Trat 5 vs Trat 6} & 0.34 & & 0.36 & & 0.58 \\
\hline \multicolumn{5}{|c|}{ Usar enzima vs No usar enzima } & 0.06 & & 0.05 & & 0.13 \\
\hline
\end{tabular}

Trat $=$ Tratamiento, GDI $=$ Grasa dorsal inicial, GDF = Grasa dorsal final, AMLI = Área del músculo longissimus inicial, AMLF = Área del músculo longissimus final, PCMI = Porcentaje de carne magra inicial, PCMF $=$ Porcentaje de carne magra final. DSST $=$ Dietas sin salvado de trigo, DCST $=$ Dietas con salvado de trigo.

lización de la energía en cerdos (Olukosi et al. 2007, Nortey et al. 2007), debido al aumento en la producción de ácidos grasos volátiles y la mayor disponibilidad de mono y polisacáridos en el intestino (van der Meulen et al. 2001).

Contrario a lo esperado, el uso de estas enzimas redujo la GDP, PVF y GCM en las dietas con salvado de trigo, resultados que coinciden con lo expresado por Reynoso-González et al. (2010) quienes encontraron que al alimentar cerdos en la etapa de crecimiento con salvado de trigo (20\%), no hubo beneficio al adicionar levaduras sobre las variables productivas. Pero la elevada cantidad de fibra redujo el consumo de alimento y la GDP.

El uso de xilanasas al aumentar potencialmente la disponibilidad de los nutrientes que se encuentran en la dieta con salvado de trigo pudo incrementar la cantidad de energía y proteína, que se reflejó en una mayor concentración de urea en plasma, en los cerdos alimentados con dietas con 
xilanasas (Cuadro 3), ya que la reducción de la digestibilidad de compuestos nitrogenados en no rumiantes se asocia con la presencia de PNA (Nortey et al. 2008); y la inclusión de xilanasas aumenta de forma indirecta la digestibilidad de aminoácidos y PC en cerdos por el incremento en el acceso de las enzimas digestivas a la proteína de la dieta (Tahir et al. 2008). Por lo que el aumento de $P C$ en la dieta, trae como consecuencia que los excesos tengan que ser eliminados, lo cual requiere una cantidad extra de energía (Figueroa et al. 2004); que pudo ser proporcionada por el aumento en la digestibilidad de la dieta con xilanasas (Ndou et al. 2015).

Al comparar las dietas con menor concentración de energía (T2 y T5), la GDF fue mayor en T2; esto debido a que el exceso de energía se canalizó para la acumulación de grasa; mientras que en T5 la energía se utilizó para la eliminación de proteína del organismo, dado a que la concentración de urea en plasma fue mayor en T5. Este fenómeno podría explicarse, a que T2 tuvo ingredientes con un menor contenido de arabinoxilanos comparado con T5, pero debido al perfil nutricional, la cantidad de nutrientes que se podría liberar en T5 es potencialmente mayor. El aumento en la cantidad de proteína y carbohidratos en el presente trabajo se reflejó en la compensación de las 75 kcal de EM en las dietas y al incremento de urea en las dietas; ya que la adición de enzimas en dietas para cerdos al destete ha mostrado aumento en la digestibilidad de la proteína y carbohidratos al incluir cereales con alto contenido de polisacáridos no almidonosos como lo son cebada, avena, trigo y arroz (Vahjen et al. 2007, Kiarie et al. 2007).

En cerdos en crecimiento y finalización, el uso de enzimas aumenta la digestibilidad de los carbohidratos, materia seca, proteína y energía en alimentos con alto contenido de hemicelulosa (O'Shea et al. 2010, Reilly et al. 2010). La eficacia de las enzimas al utilizar subproductos de trigo ricos en fibra, mejora la ganancia de peso y conversión alimenticia, además de aumentar la digestibilidad de la materia seca, proteína y energía (Emiola et al. 2009).

\section{CONCLUSIONES}

Dietas con alto contenido de salvado de trigo afectan las variables productivas, debido a la mayor cantidad de fibra en la dieta, lo que puede representar una menor disponibilidad de nutrientes. Sin embargo, esta reducción puede ser compensada con xilanasas, que aumentan la cantidad de energía disponible en la dieta. Aunque se debe considerar que el desconocimiento preciso del incremento en la disponibilidad de nutrientes trae como consecuencia un desbalance de nutrientes, lo que se refleja en un aumento de grasa en los animales.

\section{LITERATURA CITADA}

AOAC (1990) Official Methods of Analysis. Association of Analytical Chemists 15th Ed. Arlington, VA, USA. 128p.

Chaney AL, Marbach EP (1962) Modified reagents for determination of urea and ammonia. Clinical Chemistry 8: $130-132$.

CIOMS (2002) Council for International Organizations of Medical Sciences. International guiding principles for biomedical research involving animals. Geneva, Switzerland. 28p.

Emiola IA, Opapeju FO, Slominsk BA, Nyachoti CM (2009) Growth performance and nutrient digestibility in pigs fed wheat distillers dried grains with soluble-based diets supplemented with a multicarbohydrase enzyme. Journal Animal Science 87: 2315-2322.

Figueroa-Velasco JL, Cervantes-Ramírez M, Cuca-García JM, Méndez-López M (2004) Respuesta de cerdos en crecimiento y finalización a dietas con baja proteína y energía. Agrociencia 38: 383-394. 
García E (1988) Modificaciones al sistema de clasificación de Köppen (para adaptarlas a las condiciones de la República Mexicana). 4a edición. México DF. 217p.

Hassan EG, Alkareem AMA, Mustafa AMI (2008) Effect of fermentation and particle size of wheat bran on the antinutritional factors and bread quality. Pakistan Journal of Nutrition 7: 521-526

Jha $R$, Leterme $P$ (2012) Feed ingredients differing in fermentable fibre and indigestible protein content affect fermentation metabolites and faecal nitrogen excretion in growing pigs. Animal 6: 603-611.

Karl RF, McDowell LR, Miles PH, Wilkinson NS, Funk JD, Corad JH (1979) Métodos de análisis de minerales para tejidos de plantas y animales. 2a Ed. Departamento de Ciencia Animal, Universidad de Florida. Gainesville. Florida, USA. 39p.

Kiarie E, Nyachoti CM, Slominski BA, Blank G (2007) Growth performance, gastrointestinal microbial activity, and nutrient digestibility in early-weaned swine fed diets containing flaxseed and carbohydrase enzyme. Journal Animal Science 85: 2982-2993.

Martínez-Aispuro JA, Figueroa-Velasco JL, Cordero-Mora JL, Ruíz-Flores A, Sánchez-Torres-Esqueda MT, Ortega-Cerrilla ME (2015) Efecto de xilanasas en dietas a base de sorgo con diferentes niveles de energía metabolizable y proteína cruda sobre variables productivas y de la canal de cerdos en iniciación. Revista Científica FCV-LUZ 25: 123-131.

Molist F, Hermes RG, de Segura AG, Martín-Orúe SM, Gasa J, Manzanilla EG, et al. (2011) Effect and interaction between wheat bran and zinc oxide on productive performance and intestinal health in postweaning piglets. British Journal of Nutrition 105: 1592-1600.

Ndou SP, Kiarie E, Agyekum AK, Heo JM, Romero LF, Arent S, et al. (2015) Comparative efficacy of xylanases on growth performance and digestibility in growing pigs fed wheat and wheat bran-or corn and corn DDGS-based diets supplemented with phytase. Animal Feed Science and Technology 209: 230-239.

Noblet J, Le Goff G (2001) Effect of dietary fibre on the energy value of feeds for pigs. Animal Feed Science and Technology 90: 35-52.

Nortey TN, Patience JF, Simmins PH, Trottier NL, Zijlstra RT (2007) Effects of individual or combined xylanase and phytase supplementation on energy, amino acid, and phosphorus digestibility and growth performance of grower swine fed wheat-based diets containing wheat millrun. Journal Animal Science 85: 1432-1443.

Nortey TN, Patience JF, Sands JS, Trottier NL, Zijlstra RT (2008) Effects of xylanase supplementation on the apparent digestibility and digestible content of energy, amino acids, phosphorus, and calcium in wheat and wheat by-products from dry milling fed to grower pigs. Journal Animal Science 86: 3450-3464.

NPPC (1991) Procedures to evaluate market hogs. National Pork Producers Council 3rd ed. National Pork Producers Council. Des Moines, IA, USA. 16p.

NRC (2012) Nutrient requirements tables and feed ingredient composition. National Research Council Nutrient requirements of swine. 11th Ed. National Academy Press. Washington, DC. 400p.

Olukosi OA, Sands JS, Adeola O (2007) Supplementation of carbohydrases or phytase individually or in combination to diets for weanling and growing-finishing swine. Journal Animal Science 85: 1702-1711.

O'Shea CJ, Sweeny T, Lynch MB, Gahan DA, Callan JJ, O'Doherty JV (2010) Effect of $\beta$-glucans contained in barley and oat-based diets and exogenous enzyme supplementation on gastrointestinal fermentation 
Martínez- Aispuro et al. Xilanasas en dietas para cerdos en iniciación 4(10):73-80,2017

of finisher pigs and subsequent manure odor and ammonia emissions Journal Animal Science 88: 14111420.

Reilly P, Sweeney T, O'Shea C, Pierce K M, Figat S, Smith AG, et al. (2010) The effect of cereal derived beta- glucans and exogenous enzyme supplementation on intestinal microflora, nutrient digestibility, mineral metabolism and volatile fatty acid concentrations in finisher pigs. Animal Feed Science and Technology 158: 165-176.

Reynoso- González E, Cervantes- Ramírez M, Figueroa- Velasco JL, Morales- Trejo A, Araiza- Piña A, YañezHernández J (2010) Nivel de proteína, fibra y cultivo de levadura Saccharomyces cerevisiae en dietas a base de trigo para cerdos. Agrociencia 44: 753-762.

Rosenfelder P, M Eklund, R Mosenthin (2013) Nutritive value of wheat and wheat by-products in pig nutrition: A review. Animal Feed Science and Technology 185: 107-125.

SAGARPA (2001) Norma Oficial Mexicana-NOM-062-ZOO-1999: Especificaciones técnicas para la producción, cuidado y uso de los animales de laboratorio. Diario Oficial de la Federación. México, DF. 58p.

Saha B (2003) Hemicellulose conversion. Journal of Industrial Microbiology and Biotechnology 30: 279-291.

SAS (2010) The SAS system for Windows V8. Statistical Analysis System SAS 9.3 Institute, Cary, NC, USA. pp: 3891-4092.

Tahir M, Saleh F, Ohtsuka A, Hayashi K (2008) An effective combination of carbohydrases that enables reduction of dietary protein in broilers: Importance of hemicellulase. Poultry Science 87: 713-718.

van der Meulen J, Inborr J, Barker JGM (2001) Effects of cell wall degrading enzymes on carbohydrate fractions and metabolites in stomach and ileum of swine fed wheat bran based diets. Archives of Animal Nutrition 54: 101-115.

Vahjen W, Osswald T, Schäfer K, Simon O (2007) Comparison of a xylanase and a complex of non starch polysaccharide polysaccharide degrading enzymes with regard to performance and bacterial metabolism in weaned pigs. Archives of Animal Nutrition 61: 90-102.

Xu G, Baidoo SK, Johnston LJ, Bibus D, Cannon JE, Shurson GC (2010) Effects of feeding diets containing increasing content of corn distillers dried grains with soluble to grower finisher pigs on growth performance, carcass composition, and pork quality. Journal Animal Science 88: 1398-1410.

Yin YL, McEvoy JDG, Schulze H, Hennig U, Souffrant WB, McCracken KJ (2000) Apparent digestibility (ileal and overall) of nutrients and endogenous nitrogen losses in growing pigs fed wheat (var. Soissons) or its by-products without or with xylanase supplementation. Livestock Production Science 62: 119-132.

Ziemer CJ, Kerr BJ, Weber TE, Arcidiacono S, Morrison M, Ragauskas A (2012) Effects of feeding fiber fermenting bacteria to pigs on nutrient digestion, fecal output, and plasma energy metabolites. Journal Animal Science 90: 4020-4027.

Zijlstra RT, Owusu-Asiedu A, Simmins PH (2010) Future of NSP-degrading enzymes to improve nutrient utilization of co-products and gut health in pigs. Livestock Science 134: 255-257. 